\title{
AN ONLINE LEARNING APPROACH FOR QUANTIFYING PERSONALIZED THERMAL COMFORT VIA ADAPTIVE STOCHASTIC MODELING
}

\author{
Ali Ghahramani ${ }^{\mathrm{a}}$, Chao Tang ${ }^{\mathrm{b}}$, Burcin Becerik-Gerber ${ }^{\mathrm{c}}$ \\ ${ }^{\text {a }}$ PhD Student, Sonny Astani Dept. of Civil and Environmental Engineering, Viterbi School of Engineering, Univ. of \\ Southern California, KAP 217, 3620 South Vermont Ave., Los Angeles, CA 90089-2531. E-mail: \\ aghahram@usc.edu \\ ${ }^{\mathrm{b}}$ Graduate Student, Sonny Astani Dept. of Civil and Environmental Engineering, Viterbi School of Engineering, \\ Univ. of Southern California, KAP 217, 3620 South Vermont Ave., Los Angeles, CA 90089-2531. E-mail: \\ chaotang@usc.edu \\ ${ }^{\mathrm{c}}$ Associate Professor, Sonny Astani Dept. of Civil and Environmental Engineering, Viterbi School of Engineering, \\ Univ. of Southern California, KAP 224C, 3620 South Vermont Ave., Los Angeles, CA 90089-2531. E-mail: \\ becerik@usc.edu, Tel: +1 2137404383 (Corresponding Author)
}

\begin{abstract}
HVAC systems are the major energy consumers in commercial buildings in the United States. These systems are operated to provide comfortable thermal conditions for building occupants. The common practice of defining operational settings for HVAC systems is to use fixed set points, which assume occupants have static comfort requirements. However, thermal comfort has been shown to vary from person to person and also change over time due to climatic variations or acclimation. In this paper, we introduce an online learning approach for modeling and quantifying personalized thermal comfort. In this approach, we fit a probability distribution to each comfort condition (i.e., uncomfortably warm, comfortable, and uncomfortably cool) data set and define the overall comfort of an individual through combing these distributions in a Bayesian network. In order to identify comfort variations over time, Kolmogorov-Smirnov test is used on the joint probability distributions. In order to identify comfortable environmental conditions, a Bayesian optimal classifier is trained using online learning. In order to validate the approach, we collected data from 33 subjects, and an average accuracy of $70.14 \%$ and specificity of $76.74 \%$ were achieved. In practice, this approach could transform the comfort objectives to constrain functions and prevents pareto optimality problems.
\end{abstract}

Keywords: Thermal Comfort; Probabilistic Modeling; Online Learning; Adaptive Stochastic Modeling; Personalized Comfort; Commercial Buildings

\section{INTRODUCTION}

Commercial buildings are one of the largest energy consumers (18.9\% of the total energy consumption), and greenhouse emission sources (18.89\% of the $\mathrm{CO}_{2}$ emissions, and $19.59 \%$ of the total greenhouse gas emissions) in the United States [1, 2]. Heating, Ventilation, and Air Conditioning (HVAC) systems account for the largest share of the energy usage and gas emissions ( $43 \%$ of the commercial building energy consumption $[1,2]$ ). HVAC systems are primarily responsible for providing satisfactory thermal conditions and indoor air quality for building occupants. They are often operated based on the recommendations provided by the standards (e.g., ASHRAE Standard 55 (Thermal Environmental Conditions for Human Occupancy) [3] and ASHRAE Standard 62.1 (Ventilation for Acceptable Indoor Air Quality) [4]). Standards for thermal comfort conditions provide models, which estimate occupants' thermal sensations based on a few selected parameters (e.g., indoor air temperature, air humidity, clothing, etc.), which are measured through controlled experiments. Although more recent models (e.g., adaptive models) consider weather variations for estimating occupants' thermal sensations, they do not usually account for contextual factors (e.g., age, race, gender, etc.) that influence individuals' thermal comfort preferences. Thermal preferences vary from person to person, which suggests that a systematic procedure to quantify personalized preferences is needed [5]. In addition, many dynamic environmental 
and human related variables affect thermal comfort [6,7], and hence, individuals' thermal comfort ranges may change over time. This phenomenon requires models for personalized comfort models to be updated when new evidence is available (e.g., an occupant's perception of comfort or discomfort at a certain ambient condition is changed). Moreover, prior research proves that people perceive comfort in a range of environmental thermal conditions [8], which might provide an opportunity to save energy in buildings. Small thermal comfort related adjustments (e.g., adjusting the temperature set point by $1^{\circ} \mathrm{C}$ ) might have considerable impacts on the overall energy consumption [6]. In addition, it has been demonstrated that thermal comfort is the dominant factor influencing the overall satisfaction with indoor environments $[9$, 10]. Therefore, context dependent approaches to model and estimate individuals' thermal comfort preferences that enable more efficient HVAC operations can potentially help energy conservation strategies. Reducing energy consumption and gas emissions through more efficient HVAC (eHVAC) systems and building automation and control systems (BACS) is emphasized in the recent report by Mitigation of Climate Change group at the Intergovernmental Panel on Climate Change (IPCC) [11]. These systems require understanding occupants' behavioral patterns as well as their personal needs.

Human Centered thermal Comfort Identification (HCCI) approaches aim to address the challenges of context dependency in thermal comfort by differentiating individuals and independently addressing individuals' states of comfort. These efforts could be divided into two categories: (1) survey based approaches; and (2) physiological measurement based approaches. Survey based approaches aim to understand the state of mind for thermal comfort by asking humans to fill a questionnaire, while physiological measurements based approaches aim to understand the state of humans' body for thermal comfort through certain physiological measurements (e.g., heart rate, skin temperature, etc. [12-18]). Thus, real-time monitoring of building occupants' thermal comfort through HCCI approaches requires continuous data acquisition. However, continuous data acquisition from building occupants is a challenging task, and therefore, is not widely used in daily building operations. In order to address this challenge, Human Centered thermal Comfort Modeling (HCCM) approaches correlate instant comfort levels (HCCI outputs) with some other variables, such as environmental related variables (e.g., indoor air temperatures, clothing levels) [17, 18]. Thus, instead of continuous interactions with occupants (e.g., asking them to fill out a survey, taking physiological measurements), the selected correlated variables are used to estimate occupants' thermal comfort levels. Due to the difficulties and expense of monitoring all influential variables through a sensor network, and in order to achieve certainty in decision making, these models account for two categories of uncertainties [19]: (1) short-term comfort related uncertainties, caused by influence of variables, which cannot be monitored in real time on an individuals' comfort level, such as food intake, internal organs' health; and (2) long-term comfort related variations, caused by changes in weather or acclimation. From statistical point of view, short-term uncertainties result in a noise around a mean value, while long term uncertainties result in a shift in the mean value. Stochastic modeling, in contrast with deterministic modeling, integrates the above-mentioned uncertainties by defining degrees of beliefs (probabilities of occurrence) over the range of values that a decision should be made. The models used in standards (e.g., ASHRAE 55 -- thermal environmental conditions for human occupancy) [3]) cannot be categorized as HCCM approaches as they were built for a group of test subjects in a specific context (like in controlled experiments) and they are recommended to be used for occupants in other contexts. For example, PMV (predicted mean vote), as one of the most well-known models, is a statistical model that was created based on the results of the experiments conducted by Fanger in 60s [20]. The model maps few environment related parameters (e.g., indoor air temperature, indoor air humidity, etc.) to the PMV value of a group of occupants in an indoor environment [20]. In addition, the recently developed adaptive thermal comfort models $[8,21]$ are also built based on correlation analyses between seasonal variations of environmental conditions and subjects' thermal responses, and they are not considered as personalized adaptive models.

In this paper, we introduce an adaptive stochastic modeling approach for modeling personalized thermal comfort of building occupants. Our adaptive model enables determination and quantification of both the 
short-term and long-term comfort uncertainties. Our stochastic models are probability distributions in a Bayesian network that feeds into a binary Bayesian optimal classifier. In order to detect long-term variations, we implemented a sliding window based algorithm that detects significant statistical differences in comfort votes. We compared our model with other standard classification techniques by applying these techniques (including ours) on the thermal comfort data collected from 33 test subjects in regular office environments. Individuals' thermal comfort levels were collected through a participatory sensing (survey based) approach described in [22].

We describe our approach and its validation in this paper. In Section 2, a review of recent studies on the Human Centered thermal Comfort Identification (HCCI) and Human Centered thermal Comfort Modeling (HCCM) is presented. In Section 3, we introduce our stochastic approach for modeling personalized thermal comfort, as well as the model update procedures. In Section 4, the test bed buildings and experimental design are explained. In Section 5, we present the results for stochastic model validation for short-term effects, and long-term comfort variations. Section 6 demonstrates the implementation of our approach in compliance with ASHRAE 55 (thermal environmental conditions for human occupancy) [3]. In Section 7, a discussion on implementation requirements and future work are provided. Section 8 summarizes the findings and concludes the paper.

\section{HUMAN CENTERED THERMAL COMFORT IDENTIFICATION AND MODELING}

Human Centered thermal Comfort Identification (HCCI) has recently gained more attention due to (1) the inability of existing thermal comfort models to accurately estimate individuals' dynamic thermal preferences; and (2) the decreased cost of sensing infrastructure. HCCI approaches aim to understand individuals' states of comfort through direct measurements. These measurements record humans' perceptions or physiological responses to their thermal environments. Accordingly, two distinct categories of data acquisition approaches are used in literature: (1) survey based approaches, which try to quantify the perceptions; and (2) physiological measurement based approaches, which try to understand preferences based on physiological responses [17]. Survey based approaches require individuals to fill questionnaires about their thermal comfort levels. There are various questionnaire designs in literature with different scales, such as (1) the ASHRAE scale [3]; (2) the Bedford scale [23]; (3) the comfortableuncomfortable scale [24]; (4) the Human Building Interaction framework for Thermal Comfort (HBI-TC) scale [25]. Due to their distinct designs, and subjective human understanding, scales should be carefully chosen and used based on the required application (e.g., Bedford scale unlike ASHRAE scale extracts information regarding thermal acceptability) $[24,26]$. Physiological measurement based approaches [1217] are built upon the principle that physiological responses can be correlated with thermal discomfort. Therefore, monitoring the correlated measurements helps understanding when a subject is uncomfortable. If there was no evidence of thermal discomfort, these approaches reject the hypothesis that the subject is in a discomfort condition. However, there could be uncomfortable conditions (which is a state of mind [3]) that are not necessarily reflected in human physiological conditions [27]. Evidently, survey based approaches understand actual comfort levels more accurately than physiological approaches as they try to directly extract the state of mind of a person. In addition, the physiological measurement based approaches require extensive sensing of human body, which makes their applicability difficult in practice. The major challenge with both kinds of HCCI techniques is that they require continuous interactions with building occupants, which maybe intrusive over time.

To address the need for continuous monitoring of thermal comfort, Human Centered thermal Comfort Modeling (HCCM) approaches try to build statistical models through correlation analyses between the selected environmental parameters, occupant related parameters, and instant state of comfort (HCCI outputs). A data driven modeling technique that combines personalized coefficients with a general model of human body heat balance was introduced in [28]. The coefficients were estimated via minimizing a least square error function of the coefficients based on occupants' comfort votes. The authors argued that the data communicated by the occupants on a daily basis account for the adaptive changes in the model. 
In [29], a deep artificial neural network (ANN) learning technique was used for classifying environmental conditions into comfortable, uncomfortably warm, and uncomfortably cool. The ANN algorithm had 4 input layers (i.e., air temperature, radiant temperature, air flow, air humidity) and 5 hidden layers. The algorithm was trained with comfort votes from test subjects under controlled experiments. However, the time dependent variations of thermal comfort were not considered in the study. The authors in [30] developed an exergy-based approach that relates an individual's body exergy consumption rate with their assessed thermal sensations. Their results suggested that minimum body exergy consumption rate is associated with the sensation close to the thermal neutrality. In addition, they found that considering both convective and radiative heat exchange between a human body and the environment, indoor operative temperature is an appropriate measure for estimating the body exergy consumption rate. Time dependent variations were assumed to be inherently integrated in the exergy. An adaptive thermal comfort modeling technique, which uses the PMV model as a prior model, was introduced in [31]. The model calculated an adaptation coefficient, which decreases or increases the estimated PMV values. The adaptation coefficient was driven based on a field study that took into account local climate, culture, and social backgrounds. In [32], the authors developed an adaptive fuzzy-logic based algorithm that learns on-line using individuals' actions on thermostats and environmental conditions. The fuzzy sets were aligned with the desired changes to a thermostat. A multiple regression model that takes mean skin temperature and its time differential as input and predicts transient thermal sensations was introduced in [12]. Their results showed strong correlation (correlation coefficient of 0.839) for the proposed technique for predicting the sensations. Another detailed study [13] was completed on various points on human test subjects' skin to find the points, which are correlated with thermal sensations. The results showed that skin temperature gradients were more consistent with the thermal comfort condition than actual skin temperatures. In addition, wrist skin temperature was found to be a point that has the highest correlations with overall thermal comfort. In [15], the authors investigated the applicability of using individuals' heart rate as a representation of thermal sensation. In their study, the authors argued that heart rate was directly correlated with metabolic rate, and therefore it was correlated with thermal comfort. Their results showed statistically significant correlation between heart rate and thermal sensation for male test subjects with high Body Mass Index (BMI).

The majority of the above mentioned models lack the components for detecting time dependent variations (changes in time) in thermal comfort. In other words, the time dependent variations of personalized thermal comfort preferences were not mathematically studied in previous research efforts. Therefore, there is a need for a modeling technique that not only addresses the short-term comfort uncertainties but systematically detects long term preference changes without making any prior assumptions about occupant preferences. This modeling technique should be built on the data from occupants and the environment. Moreover, the internal knowledge base of a model should be updated when a new data point (i.e., comfort vote and associated environmental condition) is communicated by the occupant to take into account variations in thermal comfort.

\section{MATHEMATICAL APPROACH}

In this paper, we introduce a novel HCCM technique that adjusts its parameters in response to variations in individuals' thermal preferences. This modeling technique is a complementary mechanism for controlling HVAC systems in order to respond to occupant thermal needs [33]. We first introduce the mathematical approach for modeling comfort as a function of several variables (Section 3). A sample data point provides comfort information as a function of one or several variable(s). iln this study, we incorporated temperature as the environmental factor influencing the comfort. We also provide a discussion on how other variables can be integrated in this approach in Section 7. The data collection process for this study is explained in detail in Section 4. 


\subsection{Data Acquisition System}

The data acquisition system used in this study consists of a user interface (UI) for collecting occupants' thermal votes and a portable temperature/humidity sensor to measure occupants' local ambient conditions. The UI and sensor communicate the data to a database that can be queried any time. The closest temperature sensor readings $\left(T_{i}\right)$ to votes $\left(v_{i}\right)$ in time domain are selected. The data points denoted as $\left(v_{i}, T_{i}\right)$ are used for the next step, which is the development of the thermal comfort profiles. The UI interface was developed in [22] (Figure 1).

Figure 1. Components of the user interface and thermal preference scale used in data collection

This interface not only enables the classification of comfort and discomfort conditions, but also allows the quantification of different discomfort levels. Since the first step is to identify the comfort conditions, we transform the data collected through the UI (Figure 1) into three categorical variables: (1) uncomfortably warm (5 slider positions on the left side of the "no change" position); (2) comfortable ("no change" position); and (3) uncomfortably cool (5 slider positions on the right side of the "no change" position). The associated temperature values were also combined to three sets. Figure 2 shows the process of combining discomfort slider positions. In sum, we reduce the number of comfort labels from eleven (number of slider positions) to three to facilitate and simplify the model training process, which explained below.

Figure 2. (a) Input from the scale on the UI, (b) data transformed to three sets required by the algorithm

\subsection{Comfort modeling}

First, we transform the comfort/temperature data, collected through the UI into a parametric mathematical model. We define an Upper Limit (UL) and Lower Limit (LL) for temperatures that comfort can be realized and three probability distributions for uncomfortable and comfortable conditions (LD - Lower Distribution, MD - Middle Distribution, UD - Upper Distribution). LD, MD, and UD are the probability distribution functions defined on the uncomfortably cool, comfortable, and uncomfortably warm data points, respectively. UL for comfort temperatures is defined as the highest temperature that the user communicates a comfort vote. LL for comfort temperatures is defined as the lowest temperature that the user communicates a comfort vote. Figure 3 illustrates the different components of the model.

Figure 3. Segmentation of data based on Lower Limit (LL), Upper Limit (UL), Lower Distribution (LD), Middle Distribution (MD), and Upper Distribution (UD)

Evidently, LD and UD influence the range of environmental conditions that comfort is likely to be perceived. The higher the variance of the LD and UD, the smaller the range of comfortable environmental conditions at a certain confidence interval. We integrate the effects of different thermal comfort conditions into a single function by defining a Bayesian network (Figure 4) to combine the probability distributions over the range of temperatures that evidence suggests that comfort can potentially be perceived [LL to UL]. Bayesian network is a probabilistic graphical model (a directed acyclic graph) that represents a set of random variables and their conditional dependencies [34]. In our approach, the network 
represents the probabilistic relationships between the influential probability distributions (LD, MD, and UD) and the overall comfort.

Figure 4. Graphical representation of the Bayesian network

Overall comfort defines probability of comfort (C) and its negation ( comfort) is discomfort (DC). Following the Bayes rule, at any temperature in time $\left(T_{t}\right)$, the sum of $P\left(\operatorname{Comfort} \mid T_{t}\right)=1-$ $P\left(\right.$ Discomfort $\left.\mid T_{t}\right) . P\left(\right.$ Comfort $\left.\mid T_{t}\right)$ can be derived using the following equation:

$P\left(C \mid T_{t}\right)=\frac{P\left(M D \mid T_{t}\right)}{\omega_{1} \cdot P\left(L D \mid T_{t}\right)+\omega_{2} \cdot P\left(M D \mid T_{t}\right)+\omega_{3} \cdot P\left(U D \mid T_{t}\right)}$

Where, $C$ stands for comfort. $T_{t}$ stands for temperature at time t. $L D, M D, U D$ are the probability distributions described above. $\omega_{i}, i \in[1,2,3]$ represents the prior probabilities associated with $L D, M D$, $U D$. Bayes rule states that if $P\left(C \mid T_{t}\right)>0$ (in other words: $P\left(C \mid T_{t}\right)>P\left(D C \mid T_{t}\right)$ ), comfort has a stochastic dominance over discomfort and that temperature $T_{t}$ can be classified as comfortable. Accordingly, the following Bayes optimal classifier solves overall comfort in the Bayesian network in Figure 4:

$\operatorname{argmax} P\left(y=w \mid T_{t}\right)$

Where, $\mathrm{C}$ stands for comfort and DC stands for discomfort.

The challenge is to train the model as $P\left(C \mid T_{t}\right)$ is also a function of prior knowledge about the distribution weights. Training the model in its current form (Eq. 1) is a difficult task, because we need to estimate not only $\left(L D \mid T_{t}\right) P\left(M D \mid T_{t}\right)$, and $P\left(U D \mid T_{t}\right)$, but also $\omega_{1}, \omega_{2}, \omega_{3}$-- the weights of parent nodes (LD, MD, UD) probability distributions that generate a child node (overall comfort). The weights are prior probabilities in the Naïve Bayes formulation of Bayesian networks. In order to reduce the need for having a high number of data points for training, we slightly modify the formulation of the problem by assuming $\omega_{1}=\omega_{2}=\omega_{3}$, which states that all probability distributions influence the overall comfort with equal weights. In order to compensate for the assumption of equal prior distributions, we define a new hyper parameter, the Probability threshold $\left(P_{T}\right)$, which is used as a decision boundary to classify comfort and discomfort conditions. Accordingly, through the implementation of an online learning technique on an individual data set, the probability threshold that best classifies each person's comfort/discomfort votes can be selected (Figure 5). This modeling (classification) technique can be categorized as a Bayesian optimal classifier. The only difference to standard formulation of Bayesian optimal classifiers is that we combine the weights of parent distribution and presented it in terms of a probability threshold.

Figure 5. Probability threshold $\left(P_{T}\right)$ as a hard constraint for comfort vs. discomfort conditions

\subsection{Model fitting and parameter estimation}

In the current problem formulation, there are two variables (LL, UL), and three probability distributions (LD, MD, UD) that together describe the raw data and one threshold value $\left(P_{T}\right)$, which allows the combination of the variables. As explained earlier, we define the LL and UL to be the lowest and the highest temperatures a comfort vote gets communicated, respectively. For modeling LD, we must consider the following conditions: (1) we know the the probability of discomfort is 1 at LT and eventually 
reaches 0 (the limit is 0 ), if a user has not communicated any comfort vote below LT; and (2) the derivation of the probability distribution function at temperatures around the initial and end points should approach 0 to represent consistency of the distribution, as small changes on the end points do not influence the distribution. Therefore, we chose half normal distribution to fit to the data as its mathematical formulation inherently maintains the requirements explained above.. Other continuous probability distributions such as gamma, beta, and exponential distributions can also be used if they meet the above-mentioned criteria after the training process. The model (Eq. 3) requires a starting point (i.e., LT) and a standard deviation.

$f(x ; \sigma)=\frac{\sqrt{2}}{\sigma \sqrt{\pi}} \exp \left(-\frac{x^{2}}{2 \sigma^{2}}\right) \quad x>0$

Where $x$ is the random variable and $\sigma$ is the standard deviation of the distribution.

Given a fixed set of data and an underlying probability distribution (i.e., half normal distribution), the method of maximum likelihood maximizes the probability of the observed data under the half normal distribution assumption. Maximum-likelihood estimation selects the set of values of the model parameters that maximizes the likelihood function and gives a well-defined estimation approach in case of normal distribution $[19,35]$. Therefore, we use the method of maximum likelihood to estimate the parameter $\sigma$ of the model. The standard deviation is driven as follows:

$\hat{\sigma}_{L D}=\sqrt{\frac{1}{n_{x_{i} \geq L L, y_{i} \in U C}} \sum_{j=1}^{n_{x_{i} \geq L L, y_{i} \in U C}}\left(x_{j}-L L\right)^{2}}$

Where $n_{x_{i} \geq L L, y_{i} \in U C}$ represents the number of data points that the temperature is above the $L L$ and is labeled as $U C$ (uncomfortably cool). $x_{j}$ is the temperature of the data point that satisfies $x_{j} \geq L L, y_{j} \in$ $U C$.

Similar to the LD, UD can be modeled as a half normal distribution. The starting point is UL is in this case, and the standard deviation can be derived from the following equation:

$\hat{\sigma}_{U D}=\sqrt{\frac{1}{n_{x_{i} \leq U L, y_{i} \in U W}} \sum_{i=j}^{n_{x_{i} \leq U L, y_{i} \in U W}}\left(x_{j}-U L\right)^{2}}$

Where $n_{x_{i} \leq U L, y_{i} \in U W}$ represents the number of data points that the temperature is above the $U L$ and is labeled as uncomfortably warm $(U W) . x_{j}$ is the temperature of the data point that satisfies $x_{j} \leq U L, y_{j} \in$ $U W$.

A complete normal distribution is then used to describe to the middle distribution (MD).

$f(x ; \mu ; \sigma)=\frac{1}{\sigma \sqrt{2 \pi}} \exp \left(-\frac{(x-\mu)^{2}}{2 \sigma^{2}}\right)$

Where $x$ is the random variable, $\mu$ is the mean, and $\sigma$ is the standard deviation of the distribution. 
We use the method of maximum likelihood to estimate the parameters of the normal distribution:

$\hat{\mu}_{C}=\frac{1}{n_{y_{i} \in C}} \sum_{j=1}^{n_{y_{i} \in C}} x_{j}$

Where $n_{y_{i} \in C}$ is the number of data points that are labeled as comfortable (C), and $x_{j}$ is the temperature value that has been labeled as comfortable (C).

$\hat{\sigma}_{M D}=\sqrt{\frac{1}{n_{y_{i} \in C}} \sum_{j=1}^{n_{y_{i} \in C}}\left(x_{j}-\hat{\mu}\right)^{2}}$

Where $n_{y_{i} \in C}$ is the number of data points that are labeled as comfortable, and $x_{j}$ is the temperature value that has been labeled as comfortable $(\mathrm{C}), \hat{\mu}$ is the mean calculated from Eq. 7.

The probability threshold $\left(P_{T}\right)$ is the value of the probability that best classifies comfort votes and discomfort votes. Therefore, probability threshold is the hyper parameter of the classification algorithm and is determined through a search over different values (between 0 and 1) for finding a global optimal value each time the Bayesian Network is built on the training data. In order to implement this search, we discretized the range between 0 and 1 into [0.1 0.20 .30 .40 .50 .60 .70 .80 .9$]$. In the training stage, when the Bayesian network probability distributions are generated, we combine and then use the values in the probability threshold vector to classify the training data set. The value that best classified the training data is then chosen as the probability threshold for validation. The smaller the threshold, the higher number of false positives are realized. The higher the threshold, the higher number of false negatives are realized.

\subsection{Long term comfort variation detection}

The opportunity that the current formulation of the problem provides is the development of a single function that combines the effects of all input data and presentation of comfort as a probability distribution. Therefore, in order to detect time dependent comfort (preference) variations, we create a window (with a certain size) of data starting at the most recent data point and go backwards. We then implement the probabilistic model explained in the previous section, and implement a statistical test (i.e., Kolmogorov-Smirnov test) to detect if the joint probability distribution significantly differs from the joint probability distribution that is generated from all the data points. Kolmogorov-Smirnov test is a nonparametric test of the equality of continuous probability distributions. A Kolmogorov-Smirnov statistic quantifies a distance between the empirical distribution functions of two samples [36]. Once a statistical significant variation in the probability distribution

Figure 6. Process diagram for detecting unrepresentative data points

\section{EXPERIMENT PROCEDURE}

The data collection was completed in several offices in University of California (USC) campus buildings. Based on the Köppen climate classification [37], the climate of the area is defined as a dry-summer subtropical climate (also referred to as the Mediterranean climate). For such climates, the average 
temperature in the warm months is above $10^{\circ} \mathrm{C}$ and in the cold months is between -3 and $18{ }^{\circ} \mathrm{C}$ [37]. Areas with Mediterranean climate include lands around Mediterranean Sea, much of California (U.S.), parts of West and South Australia, southwestern part of South Africa, and part of central Chile [37]. Approximately 194.4 million people lived in lands with Mediterranean climate as of 1999 [38]. Considering the fact these lands are favorable living places, the population have had a continuous growth. The total area of lands with Mediterranean climate is about 905,000 miles^2 [38].

Each test subject was given an ID number and asked to communicate his/her votes with that specific ID number, using the UI (Figure 1). The temperature/humidity sensor used in the experiments was Aosong AM2302 temperature/humidity sensor, which has an accuracy of $\pm 0.5^{\circ} \mathrm{C}$ for temperature and $\pm 2 \% \mathrm{RH}$ (Relative Humidity) for temperature, and the resolution of $0.1^{\circ} \mathrm{C}$ for temperature and $1 \% \mathrm{RH}$ for humidity. The sensors where placed in a closed proximity (less than 1.5 meters to 2 meters) to the test subjects. Air temperature, radiation, air velocity, and humidity might have unsteady and time variant distributions in office spaces. In this study, we are benchmarking an individual's thermal preferences to a temperature sensor placed at a certain location in an office. If the sensor is in close proximity to the occupant, the modeling results can be used in other spaces with similar environmental conditions. However, if the sensor is located at a distance, the environmental conditions are likely not to be the same as the occupant's location, therefore the modeling results could be used only in that environment [39]. The test subjects included students, staff, and the faculty in the USC campus buildings. The test subjects were asked to communicate their votes while having their regular office activities in order to be representative of an actual implementation. The subjects were also asked not to communicate their votes during the first few minutes that they have arrived to their offices as we did not want the transient conditions of the environment influence the votes of the test subjects. We also asked the test subjects to communicate a maximum of 10 votes per day. Our goal was to eliminate the bias of the comfort information to a specific day or a condition. Finally, the test subjects were asked not to communicate their votes very frequently, specifically they were asked to have at least a 15-minute interval between each vote. The number of data points and the duration of data collection are presented in Table 1.

Table 1. Data acquisition details

As the first step of the research, we used analysis of variance (ANOVA) to find which variables influence thermal votes of an individual. The average of the $p$-values was 0.0139 with a standard deviation of 0.038 , which shows significant contribution of temperature to the thermal preferences. However, the average of the $p$-values with regards to humidity was 0.2913 with a standard deviation of 0.3273 , which shows considerably small contribution of humidity to the thermal preferences. Consequently, humidity does not provide significant information gain. Although the proposed probabilistic modeling approach can potentially integrate several factors, as an alternative to monitor and integrate all of the factors, we are adaptively keeping track of comfortable temperatures, which requires considerably smaller data points to train the Bayesian network.

\section{ANALYSIS OF THE RESULTS}

The online learning technique, described in Section 3, first uses a Bayesian network to parameterize and then combines the input data. Once a new data point (i.e., thermal vote and associated environmental thermal conditions) is collected, the algorithm checks whether enough data have been collected to reject previous data points that are no longer representative of the individual's preferences. Figure 7 presents the results for three sample subjects. Different colors in the graphs in the left column (Figure 7a) represent the data points segmentation based on the Kolmogorov - Smirnov test. The graphs on the right columns (Figure 7b) show the joint probability distributions of the comfort for each occupant as new data points are calculated by the algorithm. Since the figures would have been unrecognizable if we were to plot them once every single data point is analyzed, the probability distributions at 15 data point intervals are shown. 
As it can be seen in Figure 7, Kolmogorov - Smirnov test detected 1 statistical significant change in the comfort probability distributions for the test subject 1 . The probability distributions plotted for the test subject 1 also confirms the fact that thermal preferences of the test subject have not significantly changed over the course of the data collection. The temperature range with stochastic dominance of comfort (i.e., probability of comfort greater than discomfort) has been oscillating between $22.5^{\circ} \mathrm{C}$ and $24.5^{\circ} \mathrm{C}$. For the test subject 2, Kolmogorov - Smirnov test detected 2 statistical significant changes in comfort probability distributions. The temperature range with stochastic dominance of comfort was approximately between $22.5^{\circ} \mathrm{C}$ and $24{ }^{\circ} \mathrm{C}$ at the beginning of the data collection (the blue square curve). Preferences then moved forward towards warmer conditions in a way that temperature range with stochastic dominance of comfort lied approximately between $23.2^{\circ} \mathrm{C}$ and $25.5^{\circ} \mathrm{C}$ (the black circle curve), however at $3^{\text {rd }}$ significant change, the temperature range with stochastic dominance of comfort was approximately around $21.5^{\circ} \mathrm{C}$ to $23^{\circ} \mathrm{C}$ (the red cross lined curve). We detected 3 significant comfort variations for the test subject 3 . The temperature range with stochastic dominance of comfort was initially between $23.5^{\circ} \mathrm{C}$ and $24.6{ }^{\circ} \mathrm{C}$ (the blue square curve). The preferences changed toward a warmer temperature range (approximately between $24.5^{\circ} \mathrm{C}$ and $27.5^{\circ} \mathrm{C}$ (the black circle curve). At the $3^{\text {rd }}$ significant change, the temperature range with stochastic dominance of comfort approximately lied between $25^{\circ} \mathrm{C}$ and $26.5^{\circ} \mathrm{C}$ (the red cross lined curve). Preferences continued to change toward cooler conditions to around $23.9^{\circ} \mathrm{C}$ to $25.8^{\circ} \mathrm{C}$. Subject 3 was female and the duration of data collection was approximately 2 months. Subjects 1 and 2 were male subjects with 33 and 38 days of data collection duration, respectively (See Table 1). The reason for higher number of changes could be related to gender. Several other studies have also pointed that females experience thermal comfort differently due to biological reasons [40-43]. The Smirnov statistical variability test greatly help with the adaptive feature of our proposed model and improved the accuracy of the predictions.

Figure 7(a) Data points segmented based on Kolmogorov - Smirnov test; (b) overall comfort probability at 15 data points interval

In order to validate if our systematic approach provides higher accuracy for classifying comfortable vs. uncomfortable conditions, we compared the classification results of our proposed algorithm with several well-known generative and deterministic classification techniques (i.e., KNN, logistic regression, decision tree, and support vector machine (SVM) with a linear basis function). Each of the above-mentioned algorithms has one or several hyper parameter(s) that need to be tuned for providing their best performance during classification. We do not discuss the process of parameter tuning of these algorithms in this paper as there are several machine learning books/notes that describe the parameter tuning process [35]. In addition, in order to compare the results with respect to the static models, we used ASHRAE PMV-PPD model for classification. PMV's value between 0.5 and -0.5 represent $80 \%$ of the occupants to be satisfied. Therefore, using the humidity data collected by the sensor and setting the static values for other input parameters of the PMV (i.e., air flow velocity $=0.15$, clothing $=0.8$, metabolic rate $=1.1$ ), driven from ASHRAE Standard 55 tables [3], we calculated the temperatures at which the PMV value is between 0.5 and -0.5 and labeled it as comfortable. We then calculated the error based on the actual comfort votes of the test subjects. In order to unify the training process for all of the algorithms, we provided 10 data points to the algorithms, trained the model and then checked the classification results for a new $\left(11^{\text {th }}\right)$ data point. We then saved the result of the predictions, added the validation data point to the training set, and checked the accuracy for the next data point. We continued this process for all of the data points for each test subject (totaling 33 test subjects). We specifically used two measures of performance evaluation for the algorithms: (1) accuracy; and (2) specificity. Accuracy is defined as the ratio of all correct predictions divided by number of the predictions. Specificity is defined as the fraction of data points that are actually uncomfortable with a comfortable predicted classification. 
The hyper parameter probability threshold $\left(P_{T}\right)$, defined in Section 3.1, was selected based on tests of a set for different probability thresholds for the maximum achievable accuracy. The average and standard deviation of the optimal probability thresholds for each test subject are presented in Figure 8.

Figure 8. Average and standard deviation of optimal probability threshold $\left(P_{T}\right)$ across test subjects

As it can be seen in Figure 8, the values have considerable variability. The average of the probability thresholds was 0.42 with a 0.11 standard deviation across different individuals. Large standard deviation for each test subject show that the probability threshold have varied as new data points have been introduced to the algorithm. The probability threshold range selected in the classification training stage (once a new data point is received and processed by the algorithm) for all test subjects included 0.1 to 0.9 .

Table 2. Accuracy of different methods

Table 3. Specificity of different methods

As it can be seen in Tables 2 and 3, both accuracy and specificity measure of the proposed probabilistic approach were relatively higher than the other algorithms. The relatively higher accuracy of the proposed method is due to utilization of the Kolmogorov-Smirnov test, which detects statistically irrelevant points and removes these points from the Bayesian network probability distribution training sets. The large standard deviation for the accuracy of each classification technique was due to the fact that the overlap of comfortable and uncomfortable environmental conditions varied across all test subjects. The relatively smaller standard deviation of the proposed probabilistic approach across all classification techniques shows the consistency of the proposed procedure for predictions. However, the major advantage of developing a joint probability distribution for comfort is that we can specify the probability threshold as a decision boundary, which results in the definition of a closed range of temperatures that are labeled as comfortable. In Section 6, we describe how the probability threshold helps implementation of the proposed procedure in compliance with thermal comfort standards.

\section{COMPLIANCE WITH ASHRAE 55}

ASHRAE Standard 55 (Thermal environmental conditions for human occupancy) [3] uses the PMV-PPD model to define the requirements for indoor thermal conditions. The standard requires that percentage of dissatisfied people (PPD) to be less than 20\%, which implies that at least $80 \%$ of the occupants in a building to be satisfied. Based on the triangle inequality, if we set the probability threshold of our proposed approach to $80 \%$, the expected percentage of satisfied occupants would be greater than $80 \%$. Therefore, the ASHRAE standard requirements would be met. Table 4 presents the accuracy and specificity measures for the algorithm for all of the test subjects as averages. In cases where the joint probability distribution built from the Bayesian network does not exceed $80 \%$ at any environmental condition, the algorithm fails to find a range of environmental conditions that meet the standard's requirement. In such cases, we recommend to select the environmental condition that maximizes the joint probability distribution function.

Table 4. Accuracy and specificity values for the probability threshold of $80 \%$ 
As it can be seen, the accuracy results compared to Table 2 has decreased, as we have fixed the probability threshold to $80 \%$. In the previous section, we presented the results where the algorithm searched for the optimal probability threshold that best classified the comfort and uncomfortable conditions for each individual. The mean probability threshold in classification was $0.42( \pm 0.11)$. Therefore, the highest achievable accuracy was $70.14 \%$ and the specificity measure was $76.74 \%$. Setting the probability threshold to $80 \%$ resulted in an increase in the specificity measure, due to the fact that it has reduced the range of acceptable temperatures. The reduction in the range of temperatures, classified as comfortable, would decrease false classification of comfort (FP) errors and increases correct discomfort (TN) predictions. Increase in TN and reduction in FP increases the specificity value. The specificity value, which is above $80 \%$, demonstrates the compliance with the standards as it implies that the ratio of uncomfortable conditions labeled incorrectly as comfortable is less than $20 \%$.

\section{DISCUSSION}

The proposed approach has to be carefully used in cases that we do not have enough input data to parameterize the three probability distributions (LD, MD, and UD). For example, this might be an issue when the number of data points labeled as comfortable or uncomfortable is 0 . In cases where there is not enough data to populate LD and UD, but the MD is constructed, the algorithm disregards any unidentified distribution (LD or UD). If both LD and UD are not parametrized, the algorithm pursues the explained conservative routine and only classifies environmental conditions between LL and UL, as comfortable conditions. However, in a case that an individual only reports uncomfortable votes, which results in only parameterizing LD and UD, the algorithm would fail to classify any environmental condition that comfort can potentially be perceived. It is because there is not enough evidence (data points) of comfort. Therefore, we recommend to leave the algorithm to be as it is and do not make speculation of perceiving comfort in a range between uncomfortably warm and uncomfortably cool conditions. If we could collect substantial number of data points from individuals, we could have calculated the weights for each of the parent distributions individually. Accordingly, the decision boundary in this case would have been the temperatures that MD probability distribution is multiplied by the weight has stochastic dominance over summation of two other distributions multiplied with their associated votes.

The accuracy of the proposed approach differs for each individual due to the uncertainties related to lack of monitoring influential factors. In addition, the sensing device accuracy $\left( \pm 0.5^{\circ} \mathrm{C}\right)$ also influenced the overall accuracy measures of this study. Bayesian network probability distributions were defined through parameter estimation on the input data, and the error propagated through the time dependent variation detection (i.e., Kolmogorov-Smirnov test) and the classification. Consequently, the error realized in the classification was partly due to the sensor accuracy. However, since the data was measured with only one type of sensing device, we were not able to quantify how much of the error was caused by the sensor accuracy.

In this paper, as an alternative to monitoring all of the conditions, we are proposing to use air temperature in office buildings and to adaptively keep track of comfortable range of temperatures. Since HVAC systems in buildings often work with a single variable (i.e., temperature) control loop, the environment can be conditioned accordingly. However, if needed, the same mathematical procedure (i.e., Bayesian network structure introduced in Section 3.2) could be deployed to utilize the information gain from any other source. The only difference is that, the weights should be defined as a hyper parameter (instead of a single probability threshold) and the stochastic dominance of probability distributions that are related to comfortable votes should be part of the classification rule. The difficulty in this case is how to relate two variables (i.e., temperature and humidity) to the HVAC control variable (i.e., temperature setpoint). In addition, the approach uses substantially small number of input data to construct the initial Bayesian 
network (it creates an MD with less than 10 data points). However, if the input data was derived from a survey-based HCCI (similar to the data acquisition UI used for the validation of this paper), it still requires the building occupants to train the network based on their thermal preferences. Therefore, a next step of this research is to apply this methodology on other HCCI techniques (e.g., physiological measurements based techniques) and quantify personalized thermal comfort based on the physiological measurements and environmental factors. In this paper, we have developed a technique for mathematically detecting unrepresentative data points in the comfort data set of an individual. However, we did not map it to a certain change in the weather variation or change in life habits of the individual as there could be many other variables influencing it. We plan to investigate these research questions in our future research.

\section{CONCLUSIONS}

In this paper, we presented a systematic mathematical procedure to model thermal comfort as a function of variables and dynamically update the model to reflect individuals' comfort requirements in an online learning fashion. Our systematic procedure quantifies personalized thermal comfort based on the conditions that an individual perceives comfort or discomfort. This systematic procedure can be categorized as an online learning approach since it learns based on each input data point collected. In order to implement this approach, we first transform the raw data into three sets: uncomfortably warm, comfortable, and uncomfortably cool. Three probability distributions were parameterized using the method of maximum likelihood. We then defined the overall comfort of an individual through combing these distributions in a Bayesian network. The model provides probability of comfort as a function of one to several environmental variables. In order to identify comfort variations over time, KolmogorovSmirnov test was used on the joint probability distributions generated from the Bayesian network. A Bayesian optimal classifier was trained in an online learning format to identify comfortable environmental conditions. The results from implementing this procedure on the data collected from 33 test subjects showed superiority of this approach over other standard classification techniques. The average accuracy of $70.14 \%( \pm 8.20 \%)$ of and specificity of $76.74 \%( \pm 13.38 \%)$ were realized. These results were relatively higher than all other classification techniques used in this study for comparison. We finally applied the requirements for standard ASHRAE 55 to the proposed approach and demonstrated that it can be used in compliance with the standard using certain parameters. Therefore, this approach can be used to alter the conventional multi-objective (e.g., comfort and energy) optimization problems of building HVAC system and prevent pareto optimality conditions by transforming comfort objectives to constrain functions of a single optimization problem [44] while it remains in compliance with the ASHRAE 55 requirements.

\section{ACKNOWLEDGEMENTS}

This material is based upon work supported by the National Science Foundation under Grant No. 1351701. Any opinions, findings, and conclusions or recommendations expressed in this material are those of the authors and do not necessarily reflect the views of the National Science Foundation.

\section{REFERENCES}

[1] B.E.D. Book, , US Department of Energy, 2011 (2010).

[2] U.S. Energy Information Administration, Emissions of Greenhouse Gases in the United States 2009, Distribution of total U.S. greenhouse gas emissions by end-use sector, Table 3. DOE/EIA-0573(2009) (2011).

[3] A. Standard, Standard 55-2004, Thermal environmental conditions for human occupancy (2004). 
[4] A. Standard, Standard 62.1-2010 (2010). Ventilation for Acceptable Indoor Air Quality, Atlanta, GA, American Society of Heating, Refrigerating and Air-Conditioning Engineers, Inc.

[5] Y. Guan, M. Hosni, B. Jones, T. Gielda, Literature review of the advances in thermal comfort modeling, TRANSACTIONS-AMERICAN SOCIETY OF HEATING REFRIGERATING AND AIR CONDITIONING ENGINEERS. 109 (2003) 908-916.

[6] G. Jendritzky, R. de Dear, Adaptation and thermal environment, in: Biometeorology for adaptation to climate variability and change, Springer, 2009, pp. 9-32.

[7] G.S. Brager, R.J. de Dear, Thermal adaptation in the built environment: a literature review, Energy and Buildings. 27 (1998) 83-96.

[8] J.F. Nicol, M.A. Humphreys, Adaptive thermal comfort and sustainable thermal standards for buildings, Energy and Buildings. 34 (2002) 563-572.

[9] W. Cui, G. Cao, J.H. Park, Q. Ouyang, Y. Zhu, Influence of indoor air temperature on human thermal comfort, motivation and performance, Building and Environment. 68 (2013) 114-122.

[10] M. Frontczak, P. Wargocki, Literature survey on how different factors influence human comfort in indoor environments, Building and Environment. 46 (2011) 922-937.

[11] Working Group III - Mitigation of Climate Change, Climate Change 2014: Mitigation of Climate Change, Chapter 9 (2014).

[12] S. Takada, S. Matsumoto, T. Matsushita, Prediction of whole-body thermal sensation in the nonsteady state based on skin temperature, Building and Environment. 68 (2013) 123-133.

[13] J. Choi, V. Loftness, Investigation of human body skin temperatures as a bio-signal to indicate overall thermal sensations, Building and Environment. 58 (2012) 258-269.

[14] C. Huizenga, H. Zhang, E. Arens, D. Wang, Skin and core temperature response to partial-and whole-body heating and cooling, Journal of thermal biology. 29 (2004) 549-558.

[15] J. Choi, V. Loftness, D. Lee, Investigation of the possibility of the use of heart rate as a human factor for thermal sensation models, Building and Environment. 50 (2012) 165-175.

[16] K.C. Bicego, R.C. Barros, L.G. Branco, Physiology of temperature regulation: comparative aspects, Comparative Biochemistry and Physiology Part A: Molecular \& Integrative Physiology. 147 (2007) 616639.

[17] A. Auliciems, S.V. Szokolay, Thermal comfort (1997).

[18] C. Huizenga, Z. Hui, E. Arens, A model of human physiology and comfort for assessing complex thermal environments, Building and Environment. 36 (2001) 691-699.

[19] S. Russell, P. Norvig, A. Intelligence, A modern approach, Artificial Intelligence.Prentice-Hall, Egnlewood Cliffs. 25 (1995). 
[20] P.O. Fanger, Thermal comfort. Analysis and applications in environmental engineering., Thermal comfort.Analysis and applications in environmental engineering. (1970).

[21] R. De Dear, G.S. Brager, Developing an adaptive model of thermal comfort and preference, Center for the Built Environment (1998).

[22] F. Jazizadeh, F.M. Marin, B. Becerik-Gerber, A thermal preference scale for personalized comfort profile identification via participatory sensing, Building and Environment. 68 (2013) 140-149.

[23] T. Bedford, The Warmth Factor in Comfort at Work. A Physiological Study of Heating and Ventilation., Industrial Health Research Board Report.Medical Research Council (1936).

[24] Y. Zhang, R. Zhao, Overall thermal sensation, acceptability and comfort, Building and Environment. 43 (2008) 44-50.

[25] F. Jazizadeh, A. Ghahramani, B. Becerik-Gerber, T. Kichkaylo, M. Orosz, A Human-Building Interaction Framework for Personalized Thermal Comfort Driven Systems in Office Buildings, Journal of Computing in Civil Engineering (2013).

[26] I. Griffiths, P. Boyce, Performance and thermal comfort, Ergonomics. 14 (1971) 457-468.

[27] J.D. Hardy, Physiology of temperature regulation, Physiol.Rev. 41 (1961) 221.

[28] Q. Zhao, Y. Zhao, F. Wang, J. Wang, Y. Jiang, F. Zhang, A data-driven method to describe the personalized dynamic thermal comfort in ordinary office environment: From model to application, Building and Environment. 72 (2014) 309-318.

[29] W. Liu, Z. Lian, B. Zhao, A neural network evaluation model for individual thermal comfort, Energy and Buildings. 39 (2007) 1115-1122.

[30] A. Simone, J. Kolarik, T. Iwamatsu, H. Asada, M. Dovjak, L. Schellen, M. Shukuya, B.W. Olesen, A relation between calculated human body exergy consumption rate and subjectively assessed thermal sensation, Energy and Buildings. 43 (2011) 1-9.

[31] R. Yao, B. Li, J. Liu, A theoretical adaptive model of thermal comfort-Adaptive Predicted Mean Vote (aPMV), Building and Environment. 44 (2009) 2089-2096.

[32] P. Bermejo, L. Redondo, D.L. Ossa, D. Rodriguez, J. Flores, C. Urea, J.A. Gamez, J.M. Puerta, Design and simulation of a thermal comfort adaptive system based on fuzzy logic and on-line learning, Energy and Buildings. 49 (2012) 367-379.

[33] F. Jazizadeh, A. Ghahramani, B. Becerik-Gerber, T. Kichkaylo, M. Orosz, Personalized Thermal Comfort Driven Control in HVAC Operated Office Buildings.

[34] N. Friedman, D. Geiger, M. Goldszmidt, Bayesian network classifiers, Machine Learning. 29 (1997) 131-163.

[35] K.P. Murphy, Machine learning: a probabilistic perspective, MIT press, 2012. 
[36] F. James, Statistical methods in experimental physics, World Scientific Singapore, 2006.

[37] M.C. Peel, B.L. Finlayson, T.A. McMahon, Updated world map of the Köppen-Geiger climate classification, Hydrology and Earth System Sciences Discussions. 4 (2007) 439-473.

[38] P.R. Dallman, Plant life in the world's Mediterranean climates: California, Chile, South Africa, Australia, and the Mediterranean basin, Univ of California Press, 1998.

[39] Z. Yang, N. Li, B. Becerik-Gerber, M. Orosz, A systematic approach to occupancy modeling in ambient sensor-rich buildings, Simulation. 90 (2014) 960-977.

[40] S. Karjalainen, Gender differences in thermal comfort and use of thermostats in everyday thermal environments, Building and Environment. 42 (2007) 1594-1603.

[41] T.H. Karyono, Report on thermal comfort and building energy studies in Jakarta—Indonesia, Building and Environment. 35 (2000) 77-90.

[42] S. Karjalainen, Thermal comfort and gender: a literature review, Indoor air. 22 (2012) 96-109.

[43] J. Spagnolo, R. De Dear, A field study of thermal comfort in outdoor and semi-outdoor environments in subtropical Sydney Australia, Building and Environment. 38 (2003) 721-738.

[44] A. Ghahramani, F. Jazizadeh, B. Becerik-Gerber, A knowledge based approach for selecting energyaware and comfort-driven HVAC temperature set points, Energy and Buildings. 85 (2014) 536-548. 
Table 1. Data acquisition details

\begin{tabular}{|c|c|c|c|c|}
\hline Test Subject ID & Number of data points & Start date* & End date* & Duration (days) \\
\hline 1 & 170 & $11 / 02 / 2014$ & $15 / 03 / 2014$ & 33 \\
\hline 2 & 40 & $11 / 02 / 2014$ & $02 / 04 / 2014$ & 51 \\
\hline 3 & 68 & $11 / 02 / 2014$ & $02 / 04 / 2014$ & 51 \\
\hline 4 & 53 & $11 / 02 / 2014$ & $09 / 04 / 2014$ & 57 \\
\hline 5 & 73 & $11 / 02 / 2014$ & $12 / 03 / 2014$ & 29 \\
\hline 6 & 48 & $11 / 02 / 2014$ & $4 / 11 / 2014$ & 59 \\
\hline 7 & 52 & $11 / 02 / 2014$ & $27 / 03 / 2014$ & 44 \\
\hline 8 & 39 & $24 / 02 / 2014$ & $06 / 03 / 2014$ & 10 \\
\hline 9 & 202 & $27 / 02 / 2014$ & $3 / 20 / 2014$ & 21 \\
\hline 10 & 106 & $03 / 03 / 2014$ & $10 / 04 / 2014$ & 38 \\
\hline 11 & 123 & $03 / 03 / 2014$ & $23 / 03 / 2014$ & 20 \\
\hline 12 & 137 & $15 / 03 / 2014$ & $30 / 03 / 2014$ & 15 \\
\hline 13 & 105 & $24 / 03 / 2014$ & $07 / 04 / 2014$ & 14 \\
\hline 14 & 101 & $24 / 03 / 2014$ & $07 / 04 / 2014$ & 14 \\
\hline 15 & 96 & $28 / 03 / 2014$ & $17 / 04 / 2014$ & 20 \\
\hline 16 & 43 & $30 / 05 / 2014$ & $23 / 06 / 2014$ & 24 \\
\hline 17 & 35 & $20 / 06 / 2014$ & $25 / 06 / 2014$ & 5 \\
\hline 18 & 45 & $15 / 06 / 2014$ & $31 / 07 / 2014$ & 46 \\
\hline 19 & 65 & $15 / 06 / 2014$ & $31 / 07 / 2014$ & 46 \\
\hline 20 & 48 & $26 / 03 / 2014$ & $16 / 04 / 2014$ & 21 \\
\hline 21 & 36 & $11 / 03 / 2014$ & $24 / 03 / 2014$ & 13 \\
\hline 22 & 46 & $24 / 03 / 2014$ & $11 / 04 / 2014$ & 18 \\
\hline 23 & 102 & $24 / 03 / 2014$ & $30 / 03 / 2014$ & 6 \\
\hline 24 & 63 & $27 / 03 / 2014$ & $03 / 04 / 2014$ & 7 \\
\hline 25 & 48 & $22 / 03 / 2014$ & $03 / 04 / 2014$ & 12 \\
\hline 26 & 96 & $07 / 04 / 2014$ & $12 / 04 / 2014$ & 5 \\
\hline 27 & 53 & $15 / 10 / 2012$ & $15 / 11 / 2012$ & 32 \\
\hline 28 & 36 & $15 / 10 / 2012$ & $15 / 11 / 2012$ & 32 \\
\hline 29 & 120 & $15 / 10 / 2012$ & $01 / 12 / 2013$ & 90 \\
\hline 30 & 45 & $15 / 10 / 2012$ & $15 / 11 / 2012$ & 32 \\
\hline 31 & 27 & $15 / 10 / 2012$ & $15 / 11 / 2012$ & 32 \\
\hline 32 & 53 & $15 / 10 / 2012$ & $15 / 11 / 2012$ & 32 \\
\hline 33 & 19 & $15 / 10 / 2012$ & $15 / 11 / 2012$ & 32 \\
\hline
\end{tabular}

*Date format: DD/MM/YYYY 
Table 2. Accuracy of different methods

\begin{tabular}{lcc}
\hline \multicolumn{1}{c}{ Accuracy $\left(\frac{\mathrm{TP}^{*}+\mathrm{TN}^{*}}{\mathrm{TP}^{*}+\mathrm{TN}^{*}+\mathrm{F}^{*}+\mathrm{FN}^{*}}\right)$} & Average & Standard Deviation \\
\hline Proposed Probabilistic Procedure & $70.14 \%$ & $8.20 \%$ \\
K-Nearest Neighbors algorithm & $64.80 \%$ & $12.82 \%$ \\
Logistic regression & $66.67 \%$ & $13.85 \%$ \\
Decision tree & $66.29 \%$ & $13.26 \%$ \\
Support Vector Machine $(\mathrm{SVM})$ & $63.50 \%$ & $8.57 \%$ \\
ASHRAE PMV-PPD model & $56.06 \%$ & $14.47 \%$ \\
TP*: True Positive, TN*: True Negative, FP*: False Positive, $\mathrm{FN}^{*}$ : False Negative.
\end{tabular}

TP*: True Positive, TN*: True Negative, FP*: False Positive, FN*: False Negative. 
Table 3. Specificity of different methods

\begin{tabular}{lcc}
\hline \multicolumn{1}{c}{ Specificity $\left(\frac{\mathrm{TN}^{*}}{\mathrm{TP}^{*}+\mathrm{FP}^{*}}\right)$} & Average & Standard Deviation \\
\hline Proposed Probabilistic Procedure & $76.74 \%$ & $13.38 \%$ \\
K-Nearest Neighbors algorithm & $68.75 \%$ & $16.16 \%$ \\
Logistic regression & $70.37 \%$ & $27.12 \%$ \\
Decision tree & $68.47 \%$ & $18.93 \%$ \\
Support Vector Machine $(\mathrm{SVM})$ & $69.40 \%$ & $13.66 \%$ \\
ASHRAE PMV-PPD model & $69.49 \%$ & $16.82 \%$
\end{tabular}

TN*: True Negative, FP*: False Positive. 
Table 4. Accuracy and specificity values for the probability threshold of $80 \%$

\begin{tabular}{lcc}
\hline & Average & Standard Deviation \\
\hline Accuracy & $65.74 \%$ & $10.84 \%$ \\
Specificity & $83.20 \%$ & $12.11 \%$
\end{tabular}


FIGURES

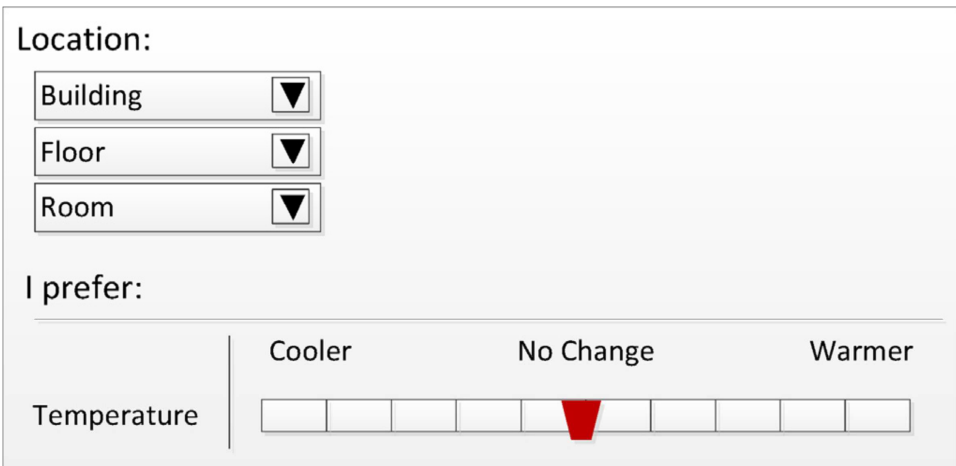

Figure 1. Components of the user interface and thermal preference scale used in data collection 


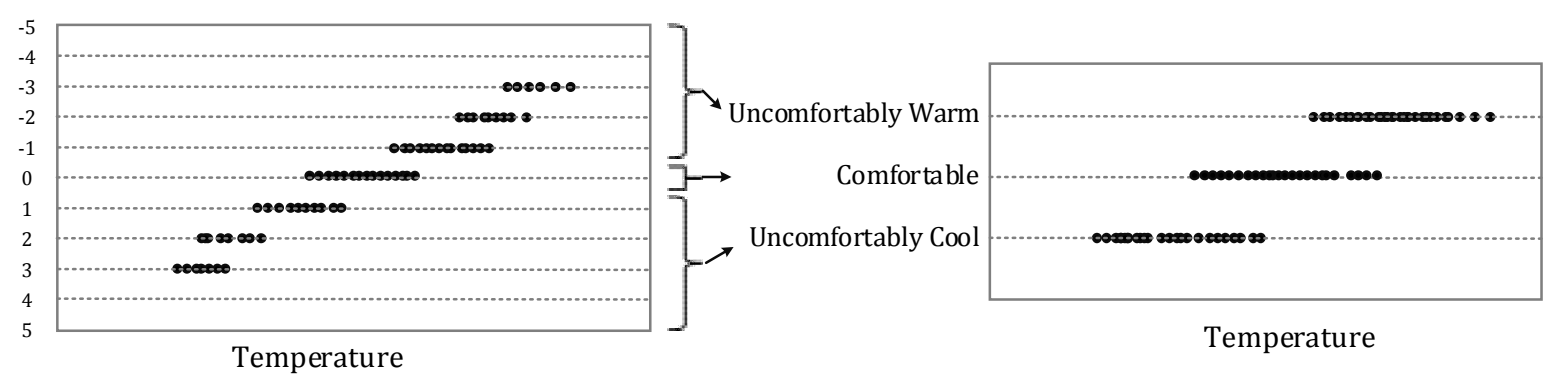

Figure 2. (a) Input from the scale on the UI, (b) data transformed to 3 sets required by the algorithm 

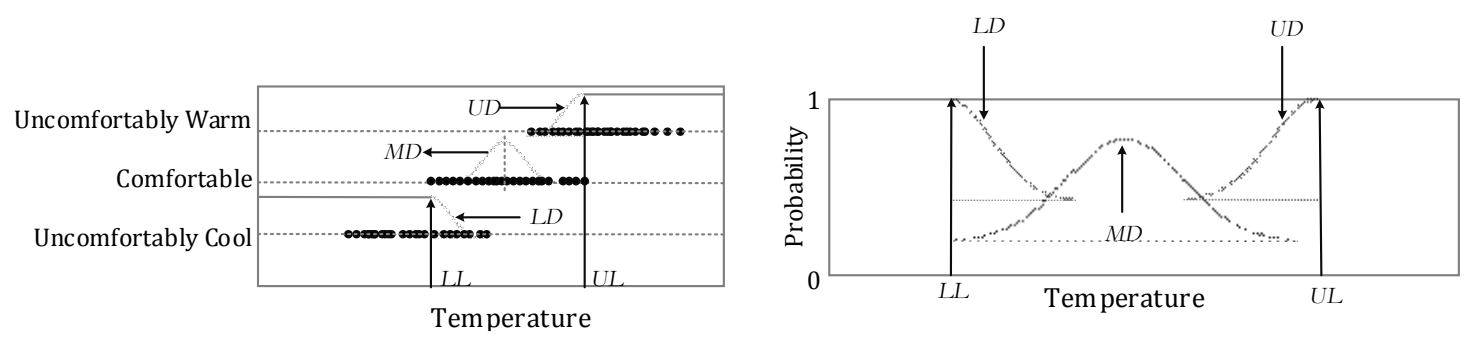

Figure 3. Segmentation of data based on Lower Limit (LL), Upper Limit (UL), Lower

Distribution (LD), Middle Distribution (MD), and Upper Distribution (UD) 


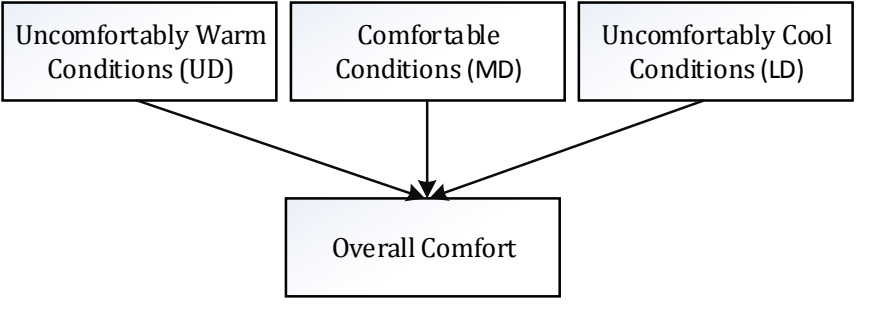

Figure 4. Graphical Representation of the Bayesian network 


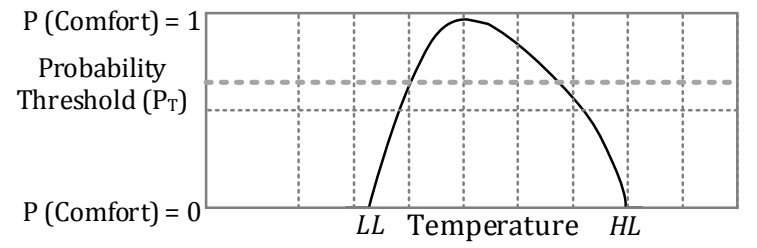

Figure 5. Probability Threshold $\left(P_{T}\right)$ as a hard constraint for comfort vs. discomfort conditions 


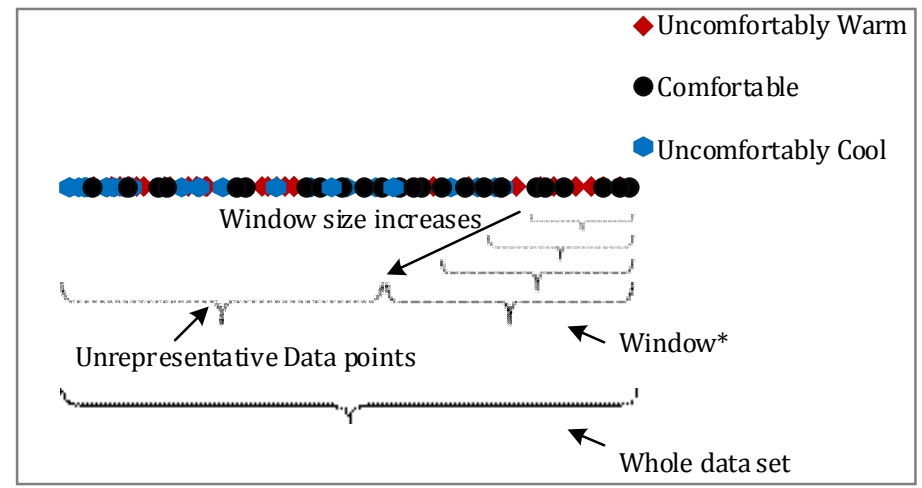

Time

Figure 6. Process diagram for detecting unrepresentative data points 

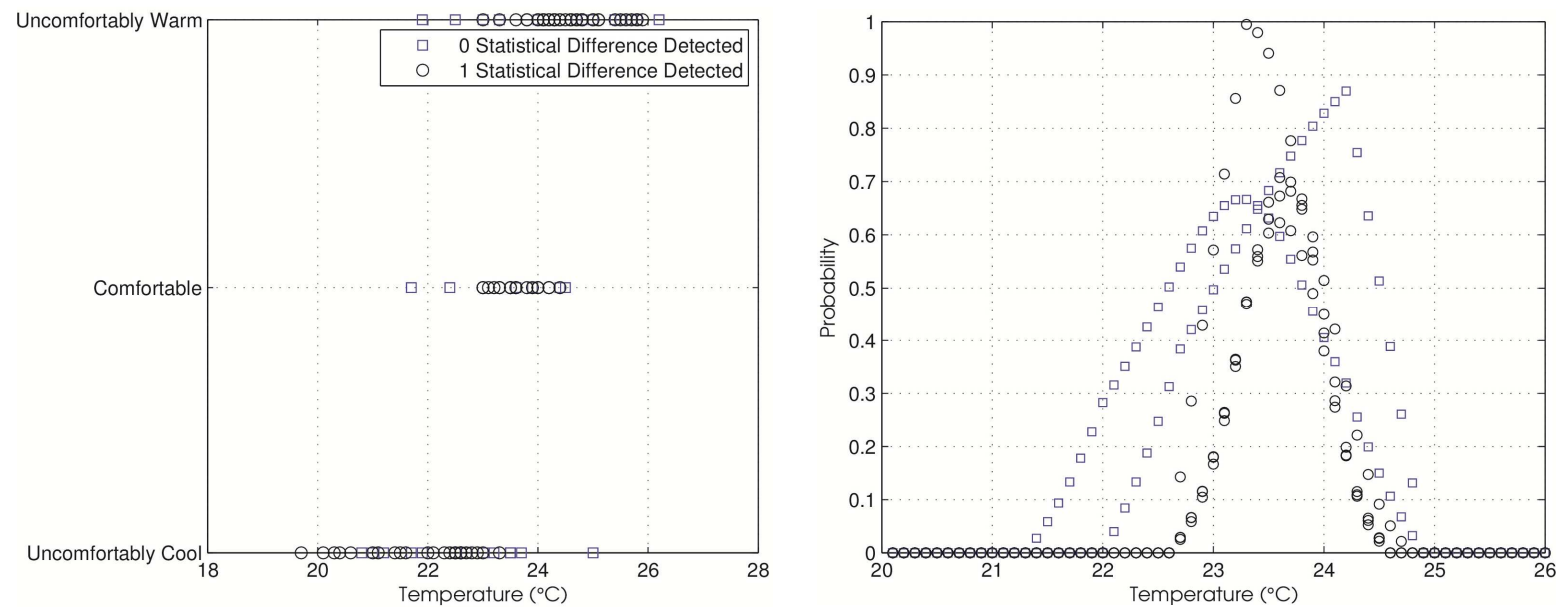

Sample Subject 1
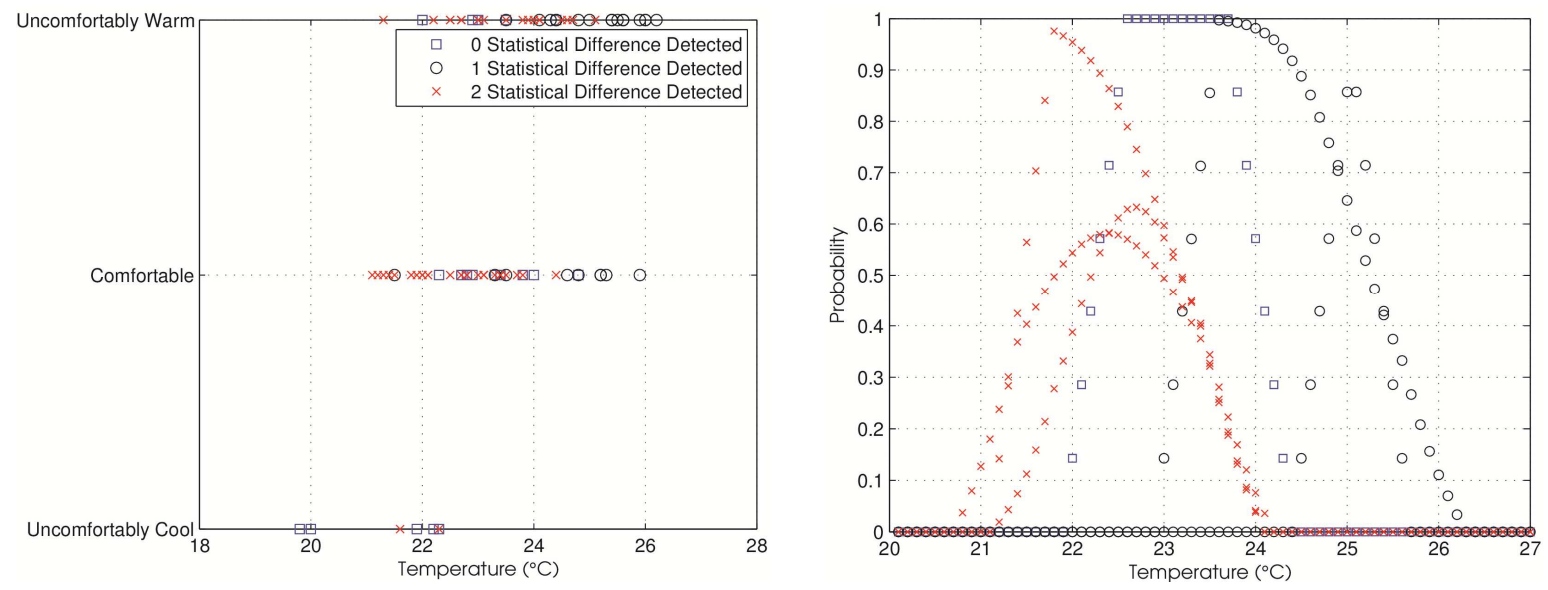

Sample Subject 2
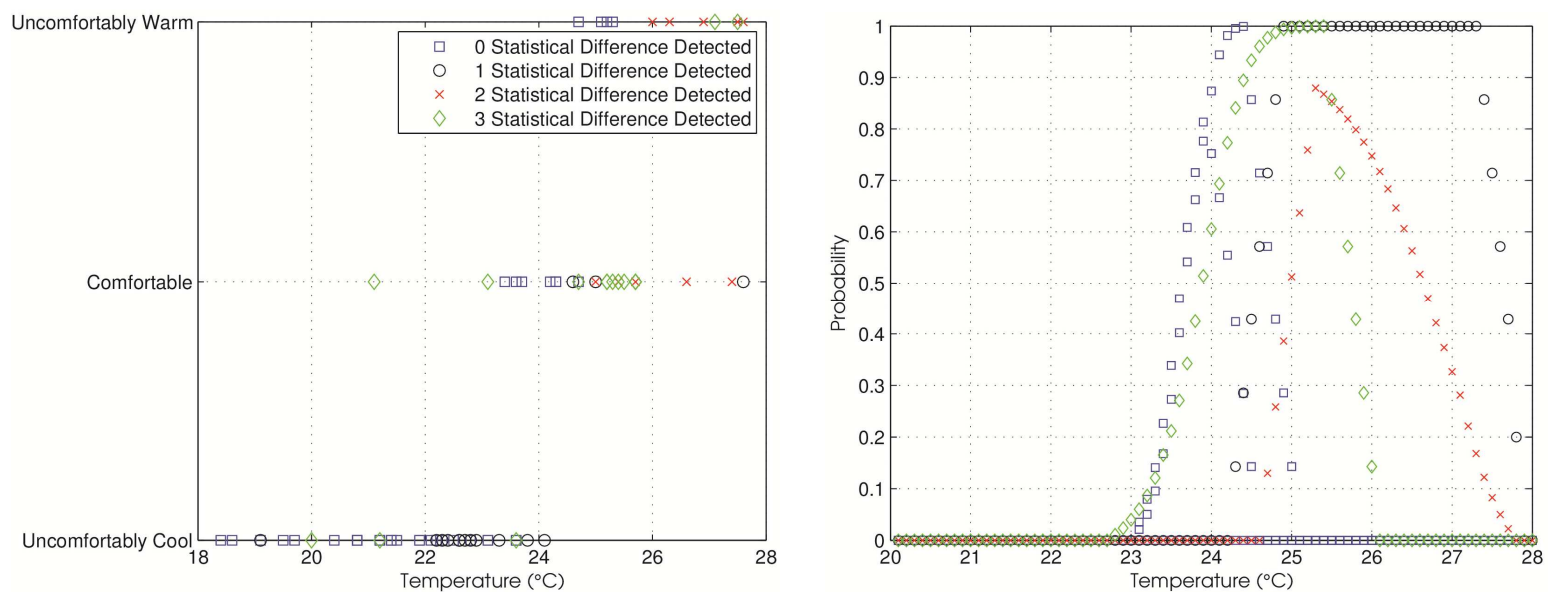

Sample Subject 3

Figure 7 (a) Data points segmented based on Kolmogorov - Smirnov test; (b) overall comfort probability at 15 data points interval 


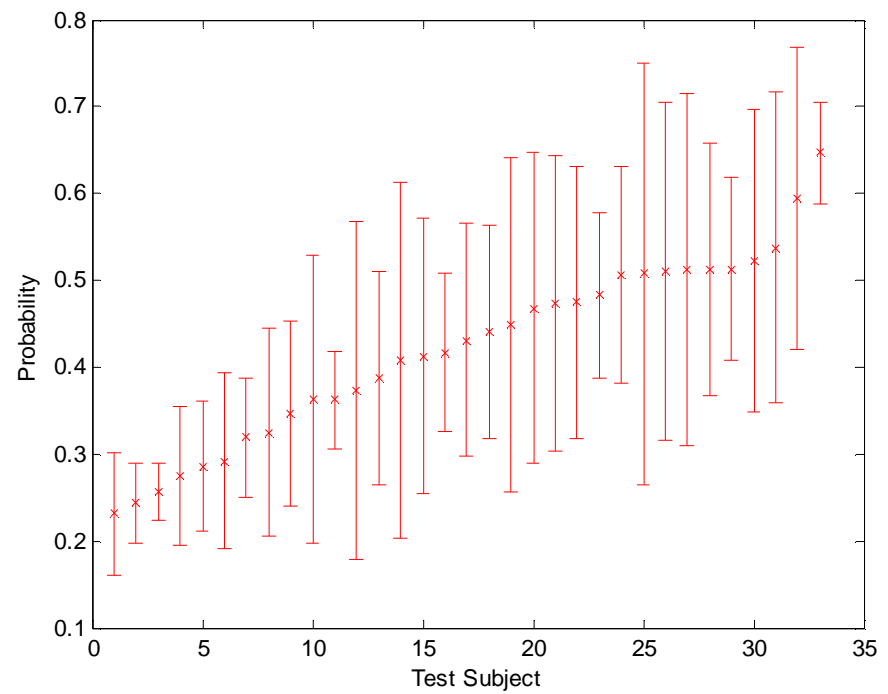

Figure 8. Average and standard deviation of optimal probability threshold $\left(P_{T}\right)$ across test subjects 